\title{
The Next Generation of Relay-Based PID Autotuners (PART 1): Some Insights on the Performance of Simple Relay-Based PID Autotuners
}

\author{
Clara M. Ionescu, Robin De Keyser \\ Ghent University, EeSA-department of Electrical energy, Systems and Automation, Technologiepark 913, 9052 Gent, Belgium \\ (ClaraMihaela.Ionescu@UGent.be ; Robain.DeKeyser@UGent.be)
}

\begin{abstract}
The paper presents theoretical insights which might lead to further development of improved relay-based PID autotuners. The analysis is based on the first generation of autotuners, namely the widely used Åström-Hägglund relay-feedback tuner. The analysis is accompanied by illustrative examples. The performance of the autotuners is evaluated against a reference PID controller which is designed using computer aided design tools and assuming full knowledge of the system's transfer function. The paper is concluded by pointing towards some ideas to design a more generally valid version of the PID autotuner.
\end{abstract}

Keywords: relay feedback, Nyquist diagram, PID controller, robustness, modulus margin

\section{INTRODUCTION}

Along the many decades in the history of control, the inventions based on feedback control had a crucial impact in the mechanical, scientific, electrical, aerospace, and information revolutions (Bernstein and Bushnell, 2002). Despite the glorious and pioneering landmarks from the past, controller design is nowadays still an art, as much as a science. Tuning controllers for optimal closed loop performance depends heavily on the process to be controlled and identification is still a burden for the control engineer and remains a significant time-consuming task.

To simplify this task, PID controllers can incorporate autotuning capabilities, which may reduce dramatically the start-up period ( $\AA$ ström and Hägglund, 1995). The autotuners are equipped with a mechanism capable of automatically computing a reasonable set of parameters when the regulator is connected to the process. Autotuning is a very desirable feature and almost every industrial PID controller provides it nowadays. These features provide easy-to-use controller tuning and have proven to be well accepted among process engineers (Leva et al., 2002).

For the automatic tuning of the PID controllers, several methods have been proposed. Åström and Hägglund ( $\AA$ ström and Hägglund, 1984; Hang et al., 1991; Åström et al., 1992) report an important and interesting approach. Their (basic) method is based on the Ziegler-Nichols frequency domain design formula (Ziegler and Nichols, 1942), but they have also described plenty of extensions. A relay connected to the process in a feedback loop is used in order to determine the critical point. Usually these preliminary tests are used to determine some (limited) information on the process model, along with the tuning of controller parameters (Schei, 1994; Scali et al., 1999).

In this contribution, we refer solely to relay based PID autotuning methods, with principles based on the beeline in the Nyquist plane.
Based on the basic Åström-Hägglund (AH) method, we present an analysis, which is also illustrated by typical examples. The performance is always compared to the 'best' PID control design, i.e. a PID tuned via computer aided design (CAD) tools, with the full knowledge of the process model.

The paper is structured as follows: the (basic) AH method and the computer aided design tool (Frequency Response toolbox) are summarized in the next section with reference to literature. An insight into the suboptimal performance of the $\mathrm{AH}$ controller for some type of processes is given in the same section. Next, a slightly different PID autotuner is presented along with theoretical insight on its suboptimal performance on illustrative examples. A final section summarizes the main outcome of this contribution and provides some ideas for the next generation of relay-based PID autotuners, namely a more generally valid controller with good performance on many process types.

\section{THE BASIC METHOD}

\subsection{Relay-based PID design: Åström-Hägglund (AH)}

Consider the example of a process $P(s)$ given by:

$$
P(s)=\frac{1}{(s+1)^{6}}
$$

of which the open loop unit step response is given in figure 1 below. As a result of the $\mathrm{AH}$ relay test - which is schematically depicted in figure 2 - the output of the process will oscillate around the setpoint and after some transient time it will enter a regime with a certain output critical amplitude $A_{c}$ and critical period $T_{c}$. The amplitude of the relay $d$ is chosen according to the amplitude of the noise in the loop; i.e. it has to provide a good signal to noise ratio. 
The typical result of such a relay experiment is depicted in figure 3. Given the oscillation amplitude, the critical gain is $K_{c}=\frac{4 d}{\pi A_{c}}$ and the controller parameters are calculated as:

$$
K_{p}=0.6 K_{c} ; \quad T_{i}=0.5 T_{c} ; \quad T_{d}=0.25 T_{i}
$$

with the (usual) choice $T_{i}=4 * T_{d}$ corresponding to a controller transfer function having 2 identical zeros.

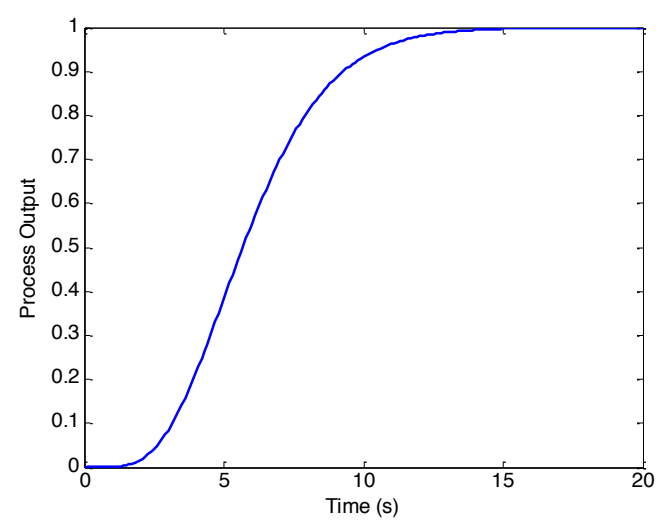

Fig. 1. Open loop unit step response for process (1).

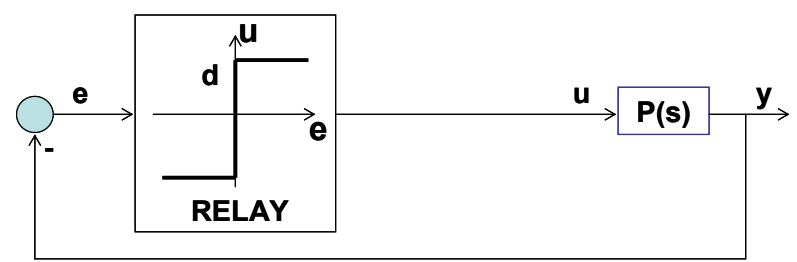

Fig. 2. Schematic representation of the relay test setup.

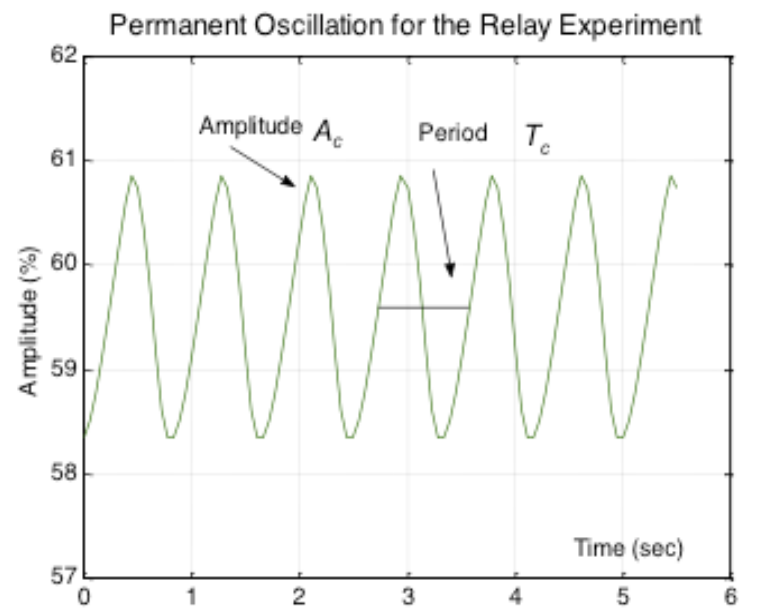

Fig. 3. An oscillatory output signal as a result of the relay test.

\subsection{A Computer Aided Design tool (FRtool)}

For comparison purposes, we use a computer aided design (CAD) tool for designing PIDs using full knowledge of the process model. In this paper, the CAD has been based on the
Frequency Response tool (FRtool) for Matlab® as described in (De Keyser and Ionescu, 2006). The reader could also use the Root Locus approach (RLtool) in Matlab ${ }^{\circledR}$ or any other model-based PID design method in order to produce a welltuned PID which will serve as reference for the autotuner evaluation.

The closed loop responses for a setpoint step (value +1$)$ at $t=0$ and for an input disturbance step (value -1 ) at $t=30$ are depicted in figure 4 for the AH PID controller and for the reference PID (designed via FRtool). As observed from this figure, both controllers perform more-or-less similarly (from a 'practical' point-of-view). More specifically: for this type of process, the autotuner does a really great job!
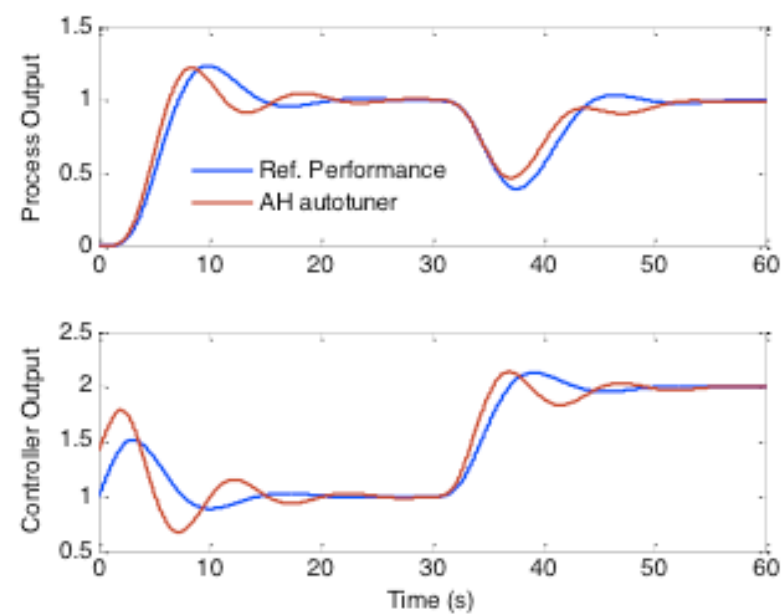

Fig. 4. Closed loop reference tracking and disturbance test for the process (1), with the AH and reference PID. AH: $K_{p}=1.41, T_{i}=5.45, T_{d}=1.36$. FRtool: $K_{p}=1, T_{i}=4, T_{d}=1$.

\subsection{Counter-example and theoretical insight}

Consider now the example of an integrating process (e.g. a positioning system) given by

$$
P(s)=\frac{32}{s(s+3)(s+21)}
$$

with the open-loop impulse response given in figure 5 .

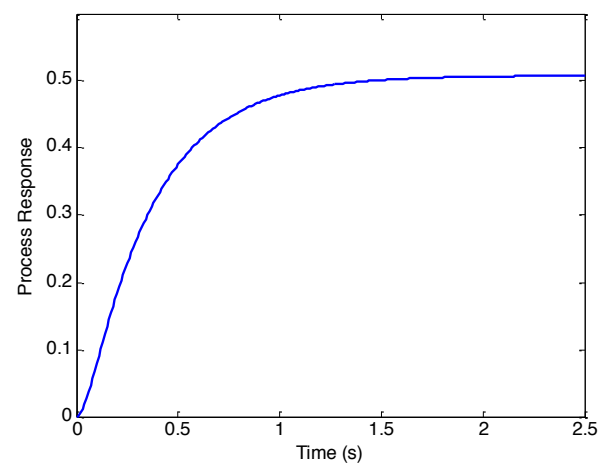

Fig. 5. Open loop unit impulse response for process (3). 
The responses for a setpoint step (of value +1 ) and for an input disturbance step (of value -20) are shown in figure 6 for the autotuned PID controller and for the reference PID (designed via FRtool). As observed from this figure, the autotuner fails to give a satisfactory performance.
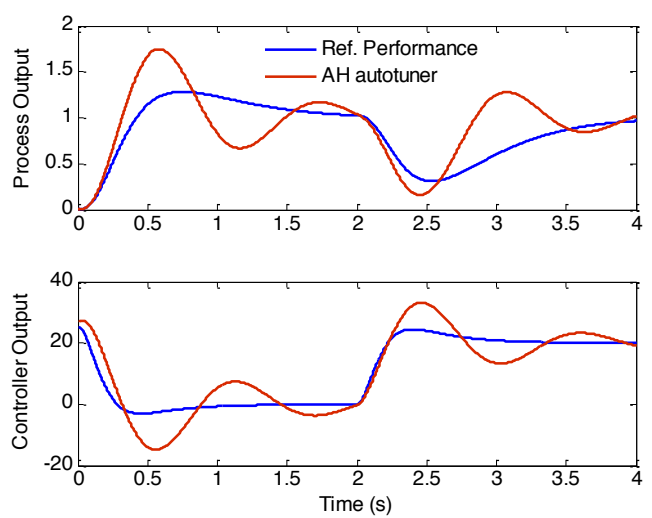

Fig. 6: Closed loop reference tracking and disturbance test for the process (3), with the AH PID and reference PID (FRtool). AH: $K_{p}=26.34, T_{i}=0.41, T_{d}=0.10$. FRtool: $K_{p}=25, T_{i}=0.8$, $T_{d}=0.2$.

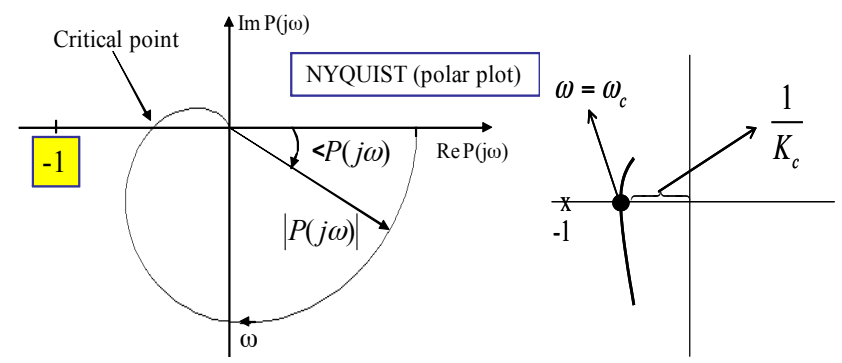

Fig. 7: The process Nyquist plot and the intersection of the beeline with the negative real axis (left) and schematic of the determined point (right)

The Åström-Hägglund autotuning method is based on identifying one point in the Nyquist plane: the intersection of the process beeline with the negative real axis (see figure 7) (Åström and Hägglund, 1995). Notice that figure 7 is a generic figure, not specifically for process (3). The critical frequency can be identified from the critical oscillation period $T_{c}: \omega_{c}=\frac{2 \pi}{T_{c}}$. The modulus and phase of the process at this critical frequency are as in figure 7 :

$$
\angle P\left(j \omega_{c}\right)=\Phi=-180^{\circ} ;\left|P\left(j \omega_{c}\right)\right|=M=\frac{\pi A_{c}}{4 d}=\frac{1}{K_{c}}
$$

and the process can be described at this critical frequency by:

$$
P\left(j \omega_{c}\right)=M e^{j \Phi}=\frac{1}{K_{c}} e^{-j 180}=\frac{1}{K_{c}}
$$

The controller is derived in its textbook form:

$$
R(s)=K_{p}\left(1+\frac{1}{T_{i} s}+T_{d} s\right)
$$

which for the critical frequency becomes:

$$
R\left(j \omega_{c}\right)=K_{p}\left[1+j\left(T_{d} \frac{2 \pi}{T_{c}} \quad \frac{1}{T_{i} \frac{2 \pi}{T_{c}}}\right)\right]
$$

and taking into account the values from (2):

$$
R\left(j \omega_{c}\right)=0.6 K_{c}\left[1+j\left(\frac{\pi}{4}-\frac{1}{\pi}\right)\right]=K_{c}(0.6+0.28 j)(8)
$$

The open loop frequency response is given by the product of the controller and the process $((8)$ and (5)):

$$
R\left(j \omega_{c}\right) P\left(j \omega_{c}\right)=K_{c}(0.6+0.28 j) \frac{-1}{K_{c}}
$$

which indicates that the loop Nyquist curve goes through the point $-0.6-0.28 j$, thus providing the robustness measure (defined here as the distance to point -1):

$$
R o_{A H}=\sqrt{(1-0.6)^{2}+0.28^{2}}=0.5
$$

Up-to-now, all these concepts are rather tutorial and they have been well described in the book (Åström and Hägglund, 1995, 2006). To understand now why the AH autotuner fails to provide a good closed loop performance for the integrating process (3), it is useful to look at the scheme depicted in figure 8 . If one calculates the location of the point given by (9) in the Nyquist plane, it follows that the controller has to introduce a phase lead of $+25^{\circ}$. In the corresponding Bode plot in figure 9, depicting process $P(\mathrm{~s})$ from (3) and the $\mathrm{AH}$ PID controller, we can indeed verify that the controller introduces a phase lead of $25^{\circ}$ at the frequency $\omega^{*}=9 \mathrm{rad} / \mathrm{s}$. From the same figure 9, one can also observe that around $\omega^{*}$ the (positive) slope of the controller-phase is higher than the (negative) slope of the process-phase. Notice that this observation is generally valid for integrating processes (not just for the specific example from (3)!). This is due to the fact that the phase of the process is $-180^{\circ}$ at $\omega^{*}$.

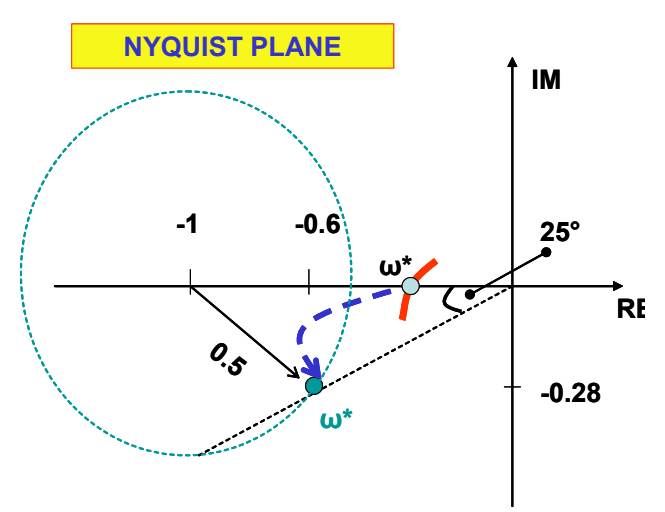

Fig. 8: Schematic representation of the AH tuning principle in the Nyquist plane, for the case of the integrating process (3). 

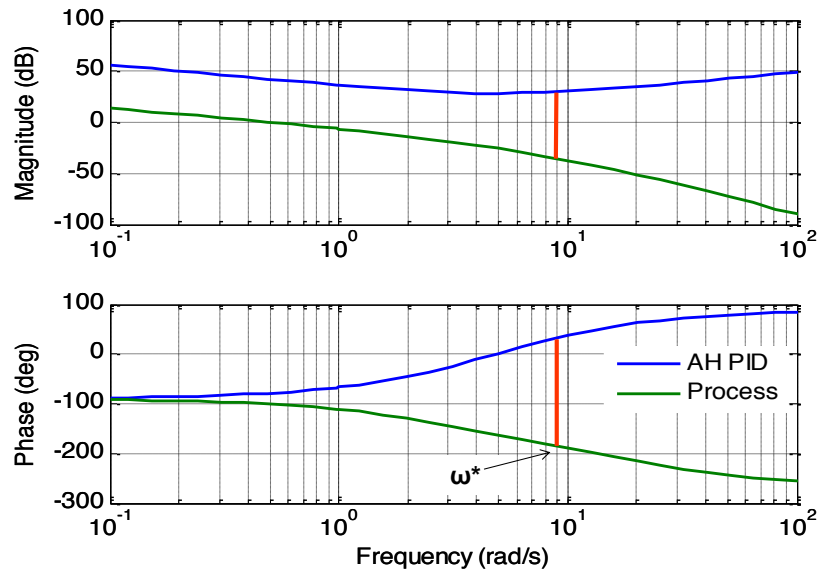

Fig. 9: The Bode plot of the process (3) and the Bode plot of the corresponding AH PID

This observation implies that at frequencies just below $\omega^{*}$, the phase of the process and controller $\left(R^{*} P\right)$ will be more negative than at $\omega^{*}$. The Nyquist curve of the loop $\left(R^{*} P\right)$ will thus cut the circle with radius 0.5 , leading to a phase margin smaller than $25^{\circ}$ and a modulus margin (distance to the point -1) less than 0.5 . Notice that in practice a usual value for the phase margin is between $40^{\circ}$ and $70^{\circ}$ and for the modulus margin between 0.5 and 0.7 .

To conclude, it is important to notice that this insight is valid for all integrating processes, not only for the example from

(3). When striving towards a generally-valid autotuner, it is therefore important to re-consider the location of $\omega^{*}$.

\section{JUST ANOTHER METHOD - NOT A BETTER ONE}

\subsection{The Phase Margin (PM) autotuner (credits also to AH)}

Similarly to the AH relay test, the PM method finds the point as expressed in (5). The task is now to find the controller parameters such that a specified phase margin is obtained (De Keyser and Ionescu, 2010). A typical phase margin is between $40^{\circ}$ and $70^{\circ}$. Generally, the larger the PM, the more robustness in the loop, less overshoot but larger settling time. Expressing that the loop frequency response should have a phase margin $P M$ at the frequency $\omega_{c}=\frac{2 \pi}{T_{c}}$ we obtain:

$$
\begin{aligned}
& R\left(j \omega_{c}\right) P\left(j \omega_{c}\right)=1 \cdot e^{j\left(-180^{\circ}+P M\right)} \\
& \cos \left(-180^{\circ}+P M\right)+j \cdot \sin \left(-180^{\circ}+P M\right)=-a-j b
\end{aligned}
$$

with $a=\cos P M$ and $b=\sin P M$, ref. figure 10 .

It follows that (11) can be re-written as:

$$
R\left(j \omega_{c}\right) P\left(j \omega_{c}\right)=-[\cos P M+j \sin P M]
$$

and equivalence of $(5)^{*}(7)$ with (12) gives us the tuning rule for $K_{p}\left(K_{p}=K_{c}{ }^{*} \cos P M\right)$ :

$$
\frac{K_{p}}{K_{c}}=\cos P M, \frac{K_{p}}{K_{c}}\left(T_{d} \omega_{c}-\frac{1}{T_{i} \omega_{c}}\right)=\sin P M
$$

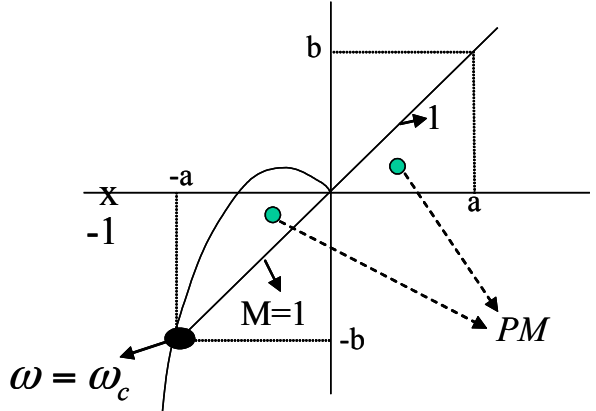

Fig. 10: Schematic of the PM principle

Choosing again $T_{i}=4 T_{d}$ and using (13), it follows that

$$
T_{d} \omega_{c}-\frac{1}{4 T_{d} \omega_{c}}=\tan P M
$$

The solution for $T_{d}$ is given by:

$$
\begin{aligned}
& T_{d} \omega_{c}=\frac{\tan P M}{2} \pm \sqrt{\left(\frac{\tan P M}{2}\right)^{2}+0.25}= \\
& \frac{\tan P M}{2} \pm \frac{\sqrt{(\tan P M)^{2}+1}}{2}=\frac{\tan P M}{2} \pm \frac{1}{2 \cos P M}
\end{aligned}
$$

Only the addition case will give a positive solution, hence:

$$
T_{d} \omega_{c}=\frac{\sin P M+1}{2 \cos P M} \rightarrow T_{d} \quad T_{c} \frac{\sin P M+1}{4 \pi \cos P M}
$$

To validate the improved performance of the PM autotuner, we test it on the integrating process (3), with a phase margin specification of $50^{\circ}$ and with the result given in figure 11 . This figure shows the remarkable performance of the PM autotuner compared to the (basic) $\mathrm{AH}$ autotuner: it gives a similar result as the PID which was designed based on the full knowledge of the process.
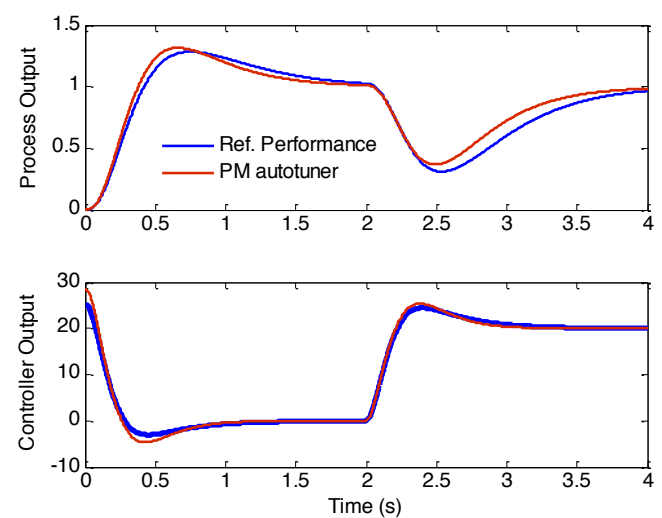

Fig. 11: Closed loop reference tracking and disturbance test for the process (3), with the auto-tuned PM PID and reference PID (FRtool). PM: $K_{p}=28.22, T_{i}=0.71, T_{d}=0.17$. FRtool: $K_{p}=25, T_{i}=0.8, T_{d}=0.2$. 


\subsection{Counter-example and theoretical insight}

Next, let us consider the following counter-example given by the process:

$$
P(s)=\frac{2}{(1+5 s)(1+10 s)} e^{-25 s}
$$

which has a significant time delay, as shown in figure 12 by its open loop step response.

The result for the PM autotuner designed for a phase margin of $70^{\circ}$ (very robust!) is given in figure 13 , compared to the reference PID designed via FRtool. It can be observed that the PM PID is unstable.

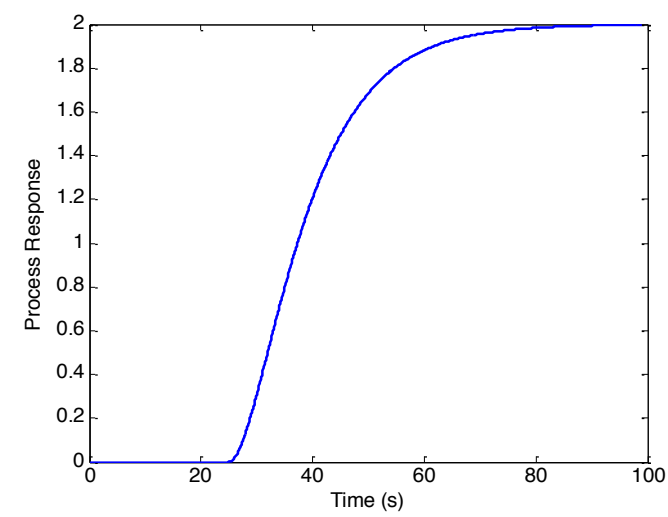

Fig. 12: Open loop unit step response for process (17)
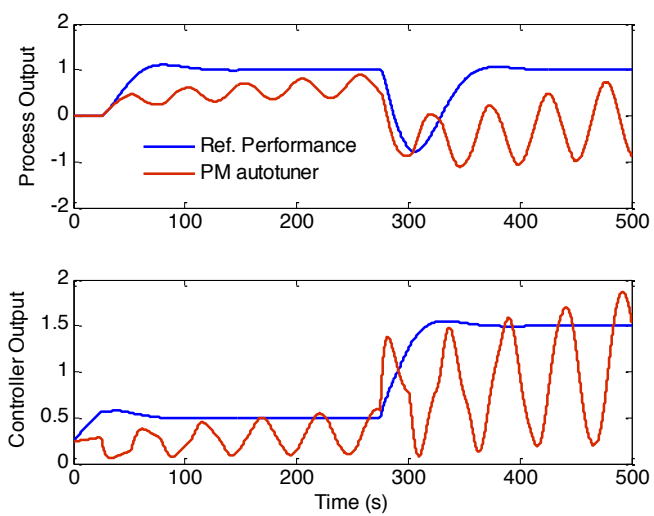

Fig. 13: Closed loop reference tracking and disturbance test for the process (17), with the PM PID and reference PID (FRtool). PM: $K_{p}=0.24, \quad T_{i}=133.58, \quad T_{d}=33.39$. FRtool: $K_{p}=0.25, T_{i}=20, T_{d}=5$.

To understand why the PM PID is resulting is such lousy performance, we have to analyze again the results in the Nyquist plane and the Bode characteristic, as previously done for the AH PID controller. If again the location is calculated of the corresponding $\omega^{*}$ point in the Nyquist plane (ref. figure 14), it follows that the controller introduces a phase lead of $+70^{\circ}$. In the corresponding Bode plot in figure 15, depicting process $P(s)$ from (17) and the PM PID controller, we can indeed verify that the controller introduces a phase lead of $70^{\circ}$ at the frequency $\omega^{*}=0.08 \mathrm{rad} / \mathrm{s}$.

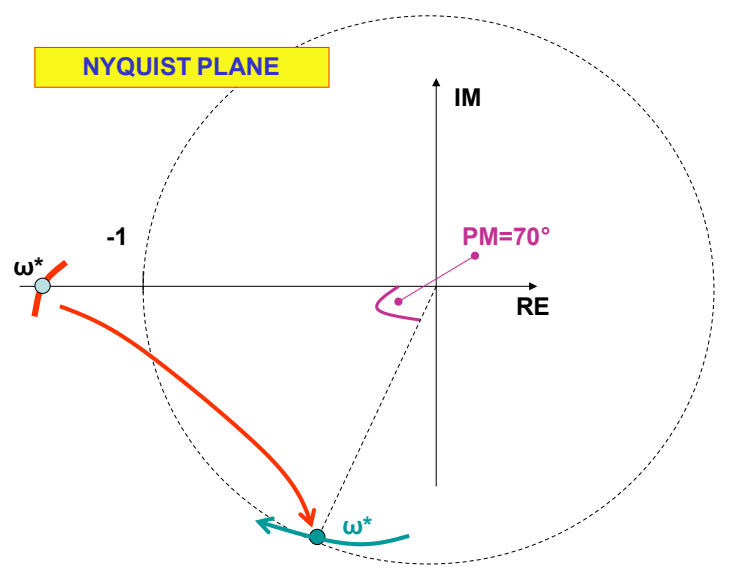

Fig. 14: Schematic representation of the PM tuning principle in the Nyquist plane, for the case of the time-delay process (17).

From the same figure 15 , one can also observe that around $\omega^{*}$ the (positive) slope of the controller-magnitude is higher than the (negative) slope of the process-magnitude. Again, this observation is generally valid for processes with considerable time-delay (i.e. not just for the specific example from (17)!). This observation implies that at frequencies higher than $\omega^{*}$, the magnitude of the process and controller $\left(P^{*} R\right)$ is increasing. As a result, the beeline of the process and controller in the Nyquist plane will go out of the unit circle, either remaining out (thus unstable closed-loop), either returning into the unit circle very close to the critical point -1 . This has indeed been verified in figure 13 for the case of the closed-loop performance of the PM controller and the process from (17), but the theoretical insight is valid for all processes with significant time delay values.
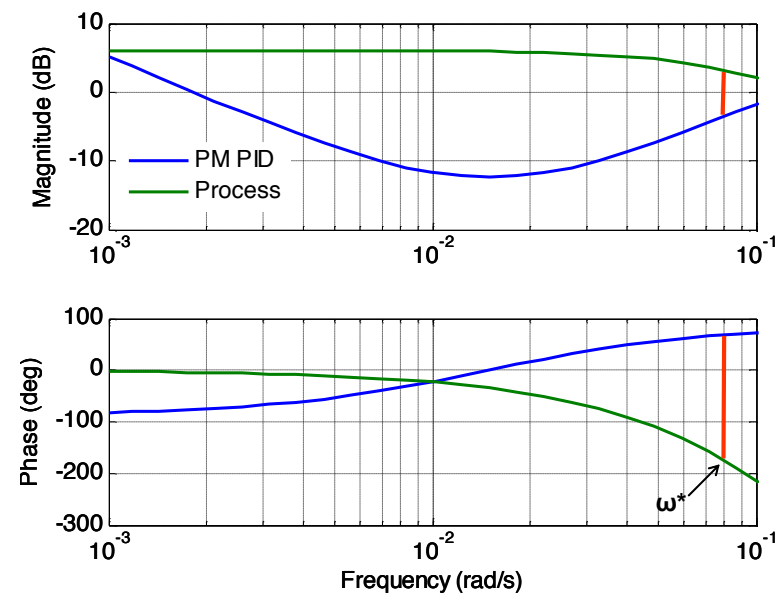

Fig. 15: The Bode plot of the process (17) and the Bode plot of the corresponding PM PID

As a second conclusion, we can state that not only the location of the point $\omega^{*}$ is important!

It is interesting to notice that the $\mathrm{AH}$ autotuner will give good results for the system (17). 


\subsection{Perspectives for a generally-valid PID relay-autotuner}

Above theoretical insights explain why well-known relaybased autotuners give good results on some processes but might fail on other processes. Based on these insights, a new relay-based autotuner has been developed which gives good performance on all processes considered above (as depicted in figures 16-18 below). Due to lack of space, the theoretical insight and the tuning rules of this novel $\mathrm{KC}$ autotuner make the subject of a related paper (De Keyser et al., 2012).
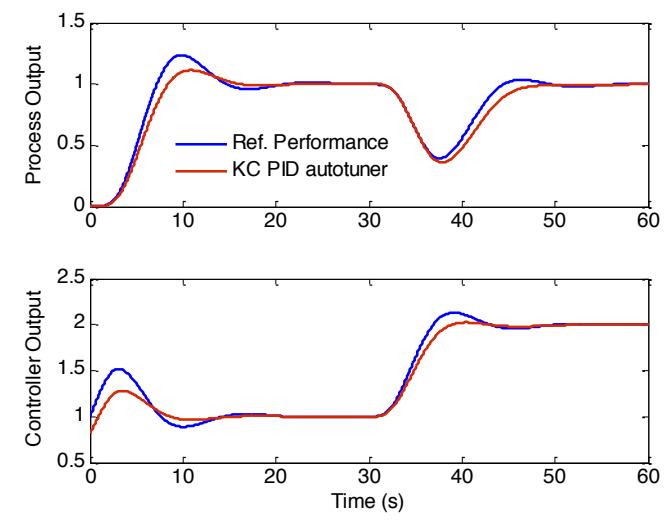

Fig. 16: The performance of the novel PID autotuner for the process (1)
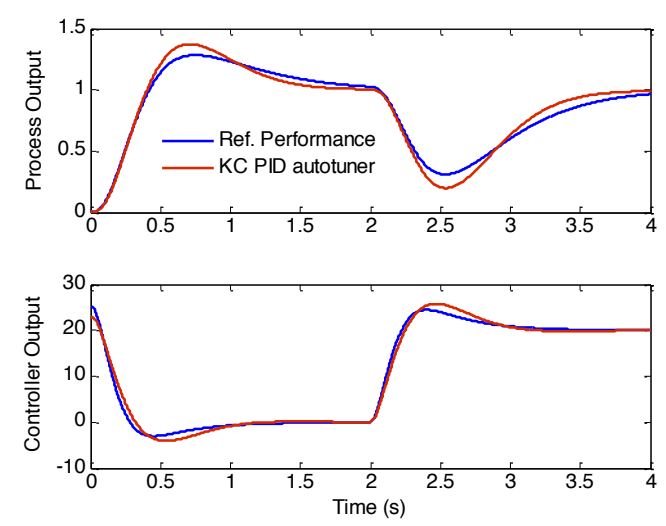

Fig. 17: The performance of the novel PID autotuner for the process (3)
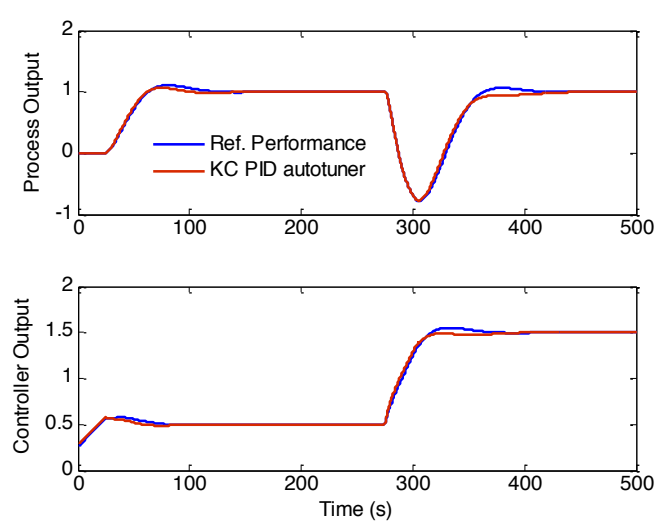

Fig. 18: The performance of the novel PID autotuner for the process (17)

\section{CONCLUSIONS}

In this paper, the dawn of a generally valid relay-based PID autotuner has been presented. Starting from the wonderfully inspiring concepts of the original Åström-Hägglund $(\mathrm{AH})$ autotuner, the road to an alternative PM autotuner has been paved by means of illustrative examples. Theoretical insights have shown the limitations of both the AH PID and the PM PID, by means of Nyquist and Bode plots. The analysis in this paper might provide an explanation on the underlying reasons for failures of the current relay-based PID autotuners. Hopefully this paper has presented some ideas to strive towards the 'utopic' autotuner, which is valid for all types of processes.

\section{REFERENCES}

Åström K.J., \& T. Hägglund. "Automatic tuning of simple regulators with specifications on phase and amplitude margins", Automatica, 20, 645-651, 1984

Åström K.J., C.C. Hang, P. Persson \& W.K. Ho. "Towards intelligent PID control". Automatica, 28, 1-9 , 1992

Åström K. J., \& T. Hägglund. "PID Controllers: Theory, Design and Tuning”. Instrument Society of America, Research Triangle Park, NC, USA, 1995

Bernstein D. \& L. Bushnell, "The history of control: from idea to technology", IEEE Control Systems Magazine, 22(2), 21-23, 2002

De Keyser R. \& C.M. Ionescu, "FRTool: a frequency response tool for CACSD in Matlab", in Proc. of the IEEE Conf. on Computer Aided Control Systems Design, Munich, Germany, 2275-2280, 2006

De Keyser R., Ionescu C., "A comparative study of three relay-based PID auto-tuners", in Proc. of the IASTED Conf. on Modelling, Identification and Control (AsiaMIC 2010), Phuket, Thailand, 303-310, 2010

De Keyser R., O.L. Joita, C.M. Ionescu, “The Next Generation of Relay-Based PID Autotuners (PART 2): A Simple Relay-Based PID Autotuner with Specified Modulus Margin", IFAC Conf. on Advances in PID Control (PID’12), Brescia Italy, 2012

Hang C.C., K.J. Åström, \& W.K., Ho, "Refinements of the Ziegler-Nichols Tuning Formula". In: IEE Proc. Design, Control Theory and Appl. 138. pp. 111-118, 1991

Leva A., C. Cox \& A. Ruano. "Hands-on Pid autotuning: a guide to better utilization". IFAC Professional Brief, 2002

Scali C., G. Marchetii, \& D. Semino, "Relay with additional delay for identification and autotuning of completely unknown processes", Ind. Eng. Chem. Res., 38, 19871997, 1999

Schei T. S., "Automatic tuning of PID controllers based on transfer function estimation", Automatica, 30, 19831986, 1994

Ziegler J. G., \& N.B., Nichols, "Optimum settings for automatic controllers". Transactions of ASME, 64, 759768,1942 